\title{
Uber die Erforschung der Lösse in der Umgebung von Brünn (Brno) in Mähren
}

\author{
Von Rudolf Musil und Karel Valoch, Mährisches Museum in Brünn.
}

Mit $1 \mathrm{Abb}$

Für die Erforschung des Eiszeitalters bildet Mähren eines der klassischen Länder. Die günstige Lage im gletscherfreien Gebiet zwischen der alpinen und der nordischen Vereisung sowie seine pfortenartige Gestaltung in den von Osten nach Westen verlaufenden Gebirgsmassen hatte zur Folge, daß dieses Land ein stetes Durchzugsgebiet und ein Bindeglied zwischen den nordöstlichen Ebenen und dem Donauraume nicht nur für den paläolithischen Menschen, sondern auch für die Tier- und Pflanzenwelt wurde. Die Zeugen dieser organischen Umwandlungen finden sich häufig in weitverbreiteten und mächtigen pleistozänen Ablagerungen, in Lössen, Flußterrassen und Höhlensedimenten.

Reiche Aufsammlungen paläolithischen und anthropologischen Materials, welche durch Ausgrabungen in vergangenen Jahrzehnten erworben wurden, bilden eine breite Basis für vergleichende Studien verschiedener Probleme des Quartärs, die an der Abteilung für Diluvium am Mährischen Museum bearbeitet werden. In vergangenen Jahren waren die Lösse und Terrassen in der Umgebung von Brünn und Wischau (Vyškov) das Ziel unserer Arbeit. Besonders im Gebiet Brünns sind die Lösse detailiert ausgebildet und bilden eine Reihe vorzüglicher Profile, in denen die gesamten Ablagerungen der vorletzten und letzten Eiszeit gut erhalten sind. Die jüngeren Lösse sind oft mit Flußterrassen verknüpt.

Bei unserer Arbeit haben wir eine möglichst große Zahl von Profilen aus einem verhältnismäßig kleinen Gebiete zwischen Brünn und Wischau (Entfernung der entlegendsten Aufschlüsse ca. $30 \mathrm{~km}$ ) herangezogen und diese durch eine komplexe Forschungsmethode bearbeitet. Wir haben uns bemüht, für einzelne Schichten wiederkehrende und ihnen eigene Merkmale aufzufinden, die es erlauben würden, diese Schichten auch dann zu erkennen, wenn nicht alle im Profil erhalten sind. Dies ist uns tatsächlich beim Vergleichen der studierten Aufschlüsse gelungen, allerdings nur für Schichten der Würmeiszeit, die am häufigsten vertreten sind. Charakteristische Zeichen einzelner Schichten sind in ca. 50 Lehmgruben vermerkt worden.

Die im folgenden kurz zusammengefaßten Darstellungen bringen die wichtigsten Ergebnisse, welche in der Gesamtstudie über die Lösse der Umgebung von Brünn ${ }^{1}$ ) und in der in Vorbereitung stehenden Arbeit über die Lösse der Wischauer Senke ausführlich behandelt sind.

Der jüngste Löß ( $W_{3}$ von Abb. 1) ist fast in allen Aufschlüssen des beschriebenen Gebietes in einer durchschnittlichen Mächtigkeit von $2-3 \mathrm{~m}$ erhalten. Von den älteren Lössen unterscheidet sich dieser auffällig durch seine dunklere gelbbraune Farbe, welche schon beim ersten Anblick des Gesamtprofils hervortritt. Bedeutend ist auch der Inhalt von Lößpuppen, die gerade in dieser Schichte von charakteristischer immer gleich kleiner Form sind, und einer größeren Menge von $\mathrm{CaCO}_{3}$-Pseudomyzelien. Auch die bekannte, für Lösse typische senkrechte Trennbarkeit ist in dieser Schichte deutlicher und besser entwickelt. Niemals haben wir da Solifluktionserscheinungen beobachtet.

1) MusiL-Valoch-NečEsaný: Pleistocenní sedimenty okolí Brna (Pleistozäne Sedimente der Umgebung von Brünn). - Anthropozoikum 4, 1954, Prag 1955. Im Druck. 
Die darunterliegende Verlehmung (Interstadial $\mathrm{W}_{2} / 3$ ) ist durch ein braunes lößartiges Sediment gebildet (Braunerde), meist von nur geringer Mächtigkeit. Durch seine blättrige Struktur, bei der sich einzelne dünne Plättchen schräg zur waagrechten Ebene lösen, und durch dunkle Färbung ist dieses vom Hangenden und Liegenden gut zu unterscheiden. Die Zahl der betreffenden Schichten, wo Solifluktionswirkungen merkbar sind, ist gering und immer durch das unmittelbar darumliegende Relief hervorgerufen, so daß man sagen kann, daß sie in diesem Gebiete diesen Horizont fast gar nicht betreffen.

Der zweitjüngste Löß $\left(W_{2}\right)$ ist in durchschnittlicher Mächtigkeit von $2 \mathrm{~m}$ erhalten und am häufigsten von periglazialen Erscheinungen betroffen. In unmittelbarer Umgebung von Brünn kann man beobachten, daß durch Solifluktion gebildete Lagen nicht nur an der Basis, sondern in einigen Fällen auch an der Oberfläche der Lößschichte entwickelt sind. Die Solifluktion wirkte daher nicht nur vor und zu Beginn der Lößbildung, sondern auch zu ihrem Ende. Dieses ist natürlich nur bei mächtigeren und gut erhaltenen Schichten ausgebildet, wogegen dort, wo sie nur einige Dezimeter betragen, eine Verschmelzung beider Solifluktionslagen eintritt. Das ist besonders gut an einzelnen Profilen nordöstlich von Brünn in der Wischauer Senke, wo das landschaftliche Relief flacher ist, zu beobachten. Hier kommt es des öfteren vor, daß das gesamte Würmprofil nur durch zwei Lösse (den jüngsten und den drittjüngsten) vertreten ist, wobei der zweitjüngste Löß entweder gänzlich fehlt oder durch mehr oder weniger mächtige Solifluktionsschichten ersetzt ist. In diesen angeführten Fällen sind also bloß zwei Lösse erhalten geblieben, und dieser Umstand dürfte, wie wir vermuten, manchen Forschern den Anlaß gegeben haben, in Landschaften mit dazu günstigen Verhältnissen nur zwei Würmlösse (Jüngerer Löß I und jüngerer Löß II) zu unterscheiden.

Die Verlehmungszone $\left(W_{1 / 2}\right)$ zwischen dem zweitjüngsten und dem drittjüngsten Löß ist immer durch eine Schwarzerde (die manchmal auch verdoppelt ist) vertreten, die gewöhnlich einen rostbraunen B-Horizont entwickelt hat. Sehr oft ist diese Schwarzerde an ihrer Oberfläche von periglazialen Wirkungen (Eiskeile, Solifluktion etc.) betroffen worden. Manchmal durchziehen sehr lange, mit Schwarzerde ausgefüllte Eiskeile die unterliegenden Horizonte, und die Oberfläche der ganzen Schicht ist durch die Solifluktion gebändert. Die beschriebenen Erscheinungen sind vor der Anwehung des nächstfolgenden Lösses entstanden, von welcher wir vermuten, daß sie erst während der zweiten Hälfte eines jeden Stadials stattgefunden hat. Diese Schicht ist die letzte pleistozäne Schwarzerde und unterscheidet sich auffällig von der schwachen braungefärbten Verlehmung des letzten Interstadials ( $W_{2} / 3$ ).

Der drittjüngste Löß $\left(W_{1}\right)$ erreicht unter den gesamten Würmlössen die relativ größte Mächtigkeit, ist hellgelber Farbe, gewöhnlich mit wenig $\mathrm{CaCO}_{3}$-Pseudomyzelien, dagegen oft mit zahlreichen Röhrchen nach verwesten Graswurzeln durchsetzt. Für die Umgebung Brünns ist dann typisch ein gegen die Basis wachsender Sandanteil, welcher in einzelne dünne Sandschichten übergeht.

Im Liegenden sind in der Brünner Umgebung, wo die Oberfläche genügend gegliedert ist, in mehreren Fällen mächtige Schwarzerden angetroffen worden (RW in Abb. 1). In einem Aufschlusse erreichen sie eine ungewöhnliche Mächtigkeit (13 m, siehe Abb. 1) und eine stark detaillierte Ausbildung der einzelnen fossilen Bodentypen. Dieser in warmen Zeitabschnitten gebildete Schichtenverband von Schwarzerden mit B-Horizonten ist ungefähr im ersten Drittel durch eine geringe Lößschichte, welche wahrscheinlich eine kalte Oszillation charakterisiert, unterbrochen. Diese stellen wir der Prae-Würm Schwankung im Sinne SoERgel's gleich. Das Vorkommen dieses außerordentlich gut erhaltenen Profils wurde durch seine günstige Lage in der Schattenseite eines Felsens ermöglicht. Eine entsprechende Lößschicht inmitten der Schwarzerdehorizonte haben wir auch in der unweit liegenden Ziegelei Malá Klajdovka gefunden. Die Oberfläche 


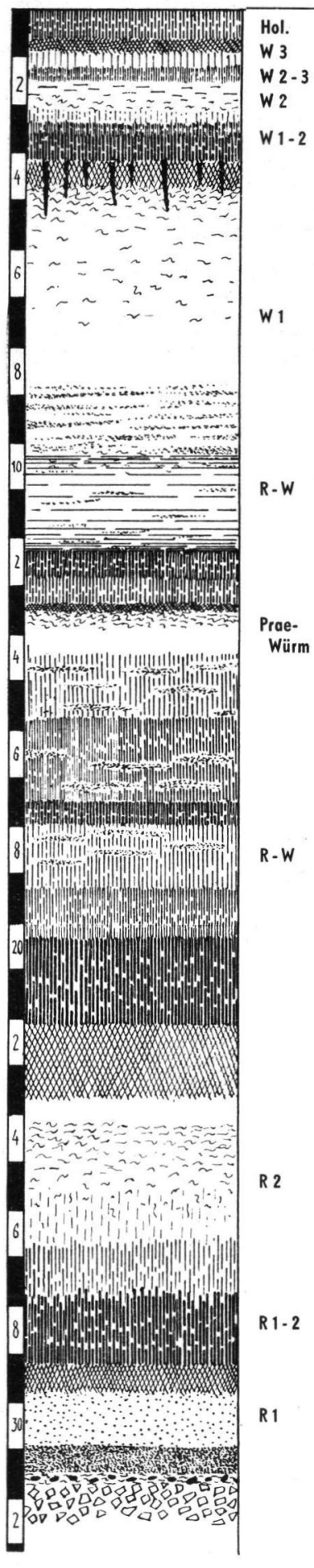

der interglazialen Sedimente ist oft durch Eiskeile zersprungen. Es ist interessant, daß ähnlich wie in den mährischen Karsthöhlen auch in der flacheren Gegend der Wischauer Senke diese interglazialen Sedimente selten erhalten sind.

Die älteren Lösse sind gegenüber den jüngeren Lössen toniger und nur bei bestimmten günstigen Verhältnissen erhalten geblieben; manchmal sind sie noch durch eine einem Interstadial entsprechende Schwarzerde getrennt.

Aus den oben angeführten Erkenntnissen geht demnach hervor, daß in diesem Gebiet die Existenz von drei jüngeren Lößschichten, an deren Basis ein mächtiger Komplex von Schwarzerden entwickelt ist, als allgemeine Erscheinung gelten kann. Die einzelnen Lößschichten sind untereinander immer durch eine Braunerde und eine tiefer liegende Schwarzerde getrennt. Daraus könnte man schließen, daß hier alle während dieser Zeit gebildeten Sedimente vollzählig erhalten sind. Auf Grund seiner Feingliederung unterscheidet W. Soergel in der letzten Eiszeit drei Kältestadien mit zwei dazwischen liegenden Warmzeiten. Die einzelnen Kältestadien werden allgemein als Zeiten der Lößbildung betrachtet, die Bildung von fossilen Böden bedeutet dagegen ihre Unterbrechung. Der im Liegenden sich befindende mächtige Komplex von Schwarzerden charakterisiert eine länger dauernde Warmperiode; auch die im ersten Interstadial gebildete Schwarzerde weist auf ein wärmeres Klima, wie dies auch in anderen $\mathrm{Ge}$ bieten konstatiert wurde. Demgegenüber wird das letzte Interstadial als viel kälter betrachtet. Diese festgestellten Erkenntnisse entsprechen vollkommen der Gliederung W. Soergel's, und deshalb benützen wir seine Bezeichnungen (Würm 1, 2, 3 usw.) für die einzelnen Schichten.

Bei der Untersuchung der erwähnten Aufschlüsse wurde den paläontologischen Resten und paläolithischen Funden große Aufmerksamkeit geschenkt. Da jedoch diese Funde nicht genügend häufig und auswertbar waren, ist es in diesem Zusammenhange nicht nötig, näher auf sie einzugehen. Die bekannten und reichen Funde von Unter-Wisternitz zeigen uns aber, daß das dortige Gravettien in der Verlehmungszone unter dem jüngsten Löß liegt, welche übereinstimmend von Fachleuten als Würm 2/3-Interstadial datiert wird. Diese Verlehmungszone entspricht unserer Braunerde zwischen dem jüngsten und zweitjüngsten Löß.

Auf der beigefügten Abbildung geben wir das besterhaltene Profil aus der Ziegelei unter dem Rosahof in Brünn wider. Die Zeichnung ist aus zwei Profilen zusammengestellt, die oberen $4 \mathrm{~m}$ entstammen der südlichen, gegenüber dem Hauptprofil liegenden Wand, wo diese Schichten besser entwickelt sind. 
Kurze Erläuterung zum Profil:

Holozän: Braunerde, unten B-Horizont

Würm 3: entkalkter verlehmter Löß

Würm 2/3: schwache Braunerde

Würm 2: kalkhaltiger Löß, im oberen Teile geschichtet

Würm 1/2: typische Schwarzerde mit B-Horizont, viele Eiskeile

Würm 1: mächtige Lößschichte, oben mit einem Karbonathorizont der vorangehenden Schicht, nach unten zu mit Sandstreifen

R-W: mächtiger Schichtenverband durch Schwarzerden, Braunerden und B-Horizonten gebildet; der oberste Teil ist in dunklen sandigen Schichten entwickelt. Unterhalb der oberen Schwarzerde ist eine dünne, leicht kalkhaltige Lößschichte eingelagert, die in der Prae-Würm Schwankung entstanden ist.

R 2: $\quad$ kalkhaltiger Löß

R 1/2: degradierte Schwarzerde, die nach oben lichter wird und allmählich in Löß übergeht

R 1: feinsandiger toniger Löß

Im Liegenden scharfkantiger, stark verwitterter Schutt.

Aus den bisherigen Erfahrungen kann man ableiten, daß die Sedimentation der einzelnen Schichten stark durch die Morphologie des Terrains beeinflußt wurde, was nicht nur in der Mächtigkeit der einzelnen gleich alten Schichten, sondern auch in der ungleichen Anzahl der Horizonte zum Ausdruck kommt. Aus diesem Grunde sind in der Umgebung von Brünn des öfteren ältere Sedimente ( $R-W$ und auch $R$ ) angetroffen worden, wogegen in der flacheren Wischauer Senke nur ausnahmsweise letzt-interglaziale Schichten erhalten sind. Lösse des zweiten Würmstadials, welche schon bei Brünn relativ gering sind, fehlen dann in der Wischauer Gegend manchmal vollkommen. Man könnte also voraussetzen, daß ähnliche Einflüsse des Terrains auch in anderen Landschaften gewirkt haben.

Manuskr. eingeg. 19. 2. 1955

Anschrift des Verf.: Dr. Rudolf Musil und Karel Valoch, Moravské Museum v Brně (Brünn), Tschechoslowakei. 